\title{
Médiévales
}

Langues, Textes, Histoire

44 | printemps 2003

Le diable en procès

\section{Le diable en procès}

\section{Martine Ostorero et Étienne Anheim}

\section{OpenEdition}

\section{Journals}

Édition électronique

URL : https://journals.openedition.org/medievales/988

DOI : 10.4000/medievales.988

ISSN : 1777-5892

\section{Éditeur}

Presses universitaires de Vincennes

\section{Édition imprimée}

Date de publication : 1 juin 2003

Pagination : 5-16

ISBN : 2-84292-142-9

ISSN : 0751-2708

\section{Référence électronique}

Martine Ostorero et Étienne Anheim, «Le diable en procès », Médiévales [En ligne], 44 | printemps 2003, mis en ligne le 02 décembre 2005, consulté le 22 avril 2022. URL : http://journals.openedition.org/ medievales/988; DOI : https://doi.org/10.4000/medievales.988

Ce document a été généré automatiquement le 22 avril 2022

Tous droits réservés 


\title{
Le diable en procès
}

\author{
Martine Ostorero et Étienne Anheim
}

1 Mettre « le diable en procès ", telle est l'ambition de ce numéro thématique portant sur la démonologie et la sorcellerie ${ }^{1}$. En cela, nous suivons les hommes de la fin du Moyen Âge et leurs chasses aux sorcières, qui donnaient lieu à l'inculpation d'individus en tant que suppôts du diable, qu'ils fussent sorciers, magiciens ou autres manipulateurs de forces démoniaques. Mais l'historien mène un procès bien plus vaste : soumettre le diable à la question, c'est l'étudier comme un pan de l'anthropologie chrétienne mais aussi s'interroger sur les conceptions médiévales des rapports entre l'homme et les forces occultes, et leurs conséquences sur les liens entre savoir et pouvoir en Occident.

Il nous a paru pertinent de commencer l'enquête au XIII ${ }^{e}$ siècle, avec l'essor des constructions institutionnelles et le développement de la pensée scolastique. La manière dont est diabolisé le catharisme dans le courant du xIII ${ }^{\mathrm{e}}$ siècle, avec l'essor de discours polémiques remplis de références à des lieux (grottes, cavernes, synagogues du diable) et à des rituels mettant en scène des adorateurs du chat diabolique, sur la base de l'étymologie catus/cathari, n'est qu'une des figures annonciatrices du futur sabbat. D'autre part nous avons souhaité, par cohérence, poursuivre jusqu'à l'orée du $\mathrm{XVII}^{\mathrm{e}}$ siècle, dépassant pour l'occasion la coupure souvent peu pertinente de 1500. Avant le XIII ${ }^{\mathrm{e}}$ siècle, le diable est craint, mais il n'est pas l'objet d'une construction juridique et théologique spécifique; après le $\mathrm{xVII}^{\mathrm{e}}$ siècle, cette construction finit par être abandonnée - entre les deux, le diable est une figure marquante de l'histoire occidentale. Dans ce vaste champ de réflexion, la recherche historique avance le plus souvent - et plutôt avec succès - par études de cas, fondées sur des textes démonologiques précis, ou s'attachant à la répression judiciaire des sorcières dans un cadre géographique et chronologique clairement délimité. Ces études permettent de mettre en lumière les enjeux spécifiques des affaires ou des textes en question et de mettre à l'épreuve les théories ou les concepts qu'ont forgés les historiens qui s'y sont intéressés, mais l'ambition d'inscrire ces éléments dans une histoire plus générale ne doit pas être abandonnée pour autant.

3 D'une part, il faut rappeler la dimension juridico-politique du «diable en procès »: l'inculpation du diable et la répression des sorciers et sorcières avec des instruments 
judiciaires particuliers tels que la procédure inquisitoire, secrète et extraordinaire, prennent place dans l'histoire de la souveraineté et de la mise en ordre de la société occidentale au sortir du Moyen Âge. D'autre part, le diable appartient à la sphère culturelle et chrétienne, parce que la théologie et le droit sont le plus souvent l'apanage des clercs, du moins à l'origine, mais aussi parce qu'il met en jeu les liens de l'homme avec le surnaturel et avec Dieu. La question diabolique émerge au moment où la société médiévale est le plus profondément christianisée sous l'égide d'une seule institution ecclésiale, après les efforts de pastorale, mais avant le Schisme et les réformes. Le monde chrétien n'a pour un temps plus de dehors : le temps des croisades est fini et les juifs commencent à être expulsés. Apparaît alors un invisible ennemi de l'intérieur : le diable, qui est l'adversaire parfait pour permettre à la société chrétienne de continuer à exister. Le problème se transforme ensuite, d'abord avec le Schisme qui lie le diable à la figure de l'adversaire religieux, puis lors de la montée des protestantismes : la présence réelle du diable est en cause, dans le processus de division entre ici-bas et au-delà qui caractérise le " désenchantement du monde ». L'attitude des XVI et XVII e siècles est très ambivalente, et montre les aménagements entre désenchantement et persistance de la croyance : comme le souligne Georg Modestin, le désenchantement n'est pas toujours le rationalisme, c'est d'abord la réorganisation des liens entre naturel et surnaturel, entre les hommes, Dieu et les démons.

Démons et sorcières : de la croyance médiévale à la critique historiographique

$4 \quad \mathrm{Au}$ point de départ de notre réflexion se trouve la volonté de comprendre le rôle de la scolastique juridique et théologique dans la répression de la sorcellerie et l'essor de la démonologie. Quelle est l'importance des usages du droit savant, en particulier dans la procédure pénale, pour la définition de ces nouvelles imputations? Quelles sont les conséquences sur le sabbat des sorcières de la démonologie héritée d'Augustin et retravaillée par la scolastique, notamment par Thomas d'Aquin? Serait-ce la démonologie thomiste qui aurait ouvert la voie aux chasses aux sorcières? Ces questions conduisent à constater l'existence d'une tension, dans les sources savantes, entre la réalité des faits incriminés et leur caractère imaginaire, tension qui se double de celle existant entre les constructions théoriques et les pratiques concrètes de la société médiévale.

5 En effet, la démonologie chrétienne ouvre, à l'égard du sabbat, un champ des possibles que les théologiens explorent jusqu'à ses limites les plus extrêmes. Pour certains théologiens $\mathrm{du} \mathrm{xv}^{\mathrm{e}}$ siècle, le vol magique des sorcières dans les airs n'est pas impossible : le démon peut agir grâce au mouvement local et transporter les corps et les objets. Il est aussi capable d'assumer ou de former artificiellement des corps, pour entrer en contact avec l'homme ; ainsi le pacte satanique, et l'ensemble des relations entre l'homme et le démon, sont possibles matériellement, comme le montre Thomas, qui fonde sur cette alliance démoniaque l'apostasie fondamentale des sorciers. Les rapports sexuels entre les démons et les sorciers, hommes ou femmes, sont également envisageables, dans la mesure où les esprits du mal prennent la forme d'incubes ou de succubes. Le démon est même capable de se fabriquer un corps qui imite toutes les propriétés de celui de l'homme et qui peut être appréhendé par la totalité des sens humains. Les démonologues décrivent le toucher ou la voix du diable ; Nicolas Jacquier va même jusqu'à décrire l'odeur du postérieur du diable comme preuve de la réalité du baiser diabolique et de la présence réelle et corporelle du diable. Quasiment tout est 
donc possible et pensable rationnellement dans le cadre de la démonologie chrétienne issue principalement d'Augustin et de Thomas, et par conséquent peut être vrai et réel.

6 Mais d'un autre côté le diable est un grand illusionniste, puisque les corps assumés par les démons ne sont que des simulacres, et non de vrais corps d'hommes, créatures divines. Et surtout il trompe l'être humain, car il peut aussi instiller des rêves et des visions dans son esprit, et agir sur sa faculté de penser : le diable lui-même induirait les hommes à penser que le sabbat n'est qu'un rêve inspiré par lui. C'est bien là le paradoxe : les pouvoirs du démon peuvent rendre le sabbat réel ou illusoire; ces deux notions différentes du diable coexistent au sein d'un même système de croyance, mais elles aboutissent à des conclusions radicalement opposées.

7 L'équivoque intellectuelle du sabbat, entre imaginaire et réalité, laisse ainsi un espace de liberté aux pratiques des théologiens et des juristes. Puisque tout est possible et pensable, le sabbat devient une affaire de croyance, à propos de laquelle les théologiens confrontent leurs opinions, de même que les juristes et les juges, laïcs ou ecclésiastiques. La procédure inquisitoire, parce qu'elle accorde une place centrale aux aveux des accusés, pose le problème de la vérité du sabbat - car ce qui était dit sous la torture était considéré comme véridique par les juges, et l'historien doit affronter à son tour cette difficulté.

8 Ces pratiques ont aussi pour effet de modifier l'équilibre de l'ensemble des savoirs médiévaux. En partant de la sorcellerie et de la répression judiciaire des sorcières, théologiens et juristes médiévaux finissent par incriminer une pratique courante, la magie. Il faut alors prendre en compte le débat médiéval sur la magie naturelle ou démoniaque, pour autant que la distinction théorique puisse se faire, les deux formes étant souvent intimement liées. Considère-t-on la magie comme une opération qui vise uniquement à utiliser les forces de la nature (pierres, astres, plantes, etc.) pour obtenir un effet particulier, ou comme une opération résultant d'une alliance passée avec le démon que l'on invoque pour obtenir un résultat précis, voire encore comme une combinaison de l'une et de l'autre? Quelles sont les conséquences de cette réflexion au niveau de la qualification de la magie rituelle, puisque celle-ci est redéfinie dans le cadre de l'hérésie, ce qui permet une action judiciaire? La plupart des traités de démonologie ne se limitent pas au problème des sorciers et de leur répression, mais évoquent ces questions touchant à la magie, en s'interrogeant sur son efficacité, son origine et ses liens avec les démons: des pratiques plus anciennes sont ainsi " contaminées " par de nouveaux savoirs produits lors de la mise en accusation des sorcières à la fin du Moyen Âge.

9 Le problème de la sorcellerie doit enfin être replacé dans les débats de méthode auxquels il a donné lieu dans les décennies récentes. Depuis les travaux pionniers de Hugh Trevor-Roper, Julio Caro Baroja, Norman Cohn, Richard Kieckefer et Robert Muchembled, qui ont permis chacun à leur manière de défricher des champs particuliers de la sorcellerie, deux grandes tendances peuvent être schématiquement distinguées: l'une s'oriente vers un traitement anthropologique des sources, l'autre vers un traitement technique et juridique, les oppositions entre les deux points de vue portant sur la réalité des faits évoquées lors des procès, en particulier sur la part relative prise par la procédure juridique dans la construction et l'aveu des récits de sabbat, par rapport à de possibles pratiques populaires. Nous avons essayé d'adopter une voie moyenne et de présenter différentes approches : s'il est probable que le sabbat est au départ une construction savante et imaginaire, celle-ci n'en a pas moins des 
effets sur le réel, y compris dans les pratiques. De même, il est certain que le sabbat s'est élaboré à partir d'éléments préexistants mais la signification de ceux-ci s'est probablement modifiée au contact de cette nouvelle formulation. Dans cette optique, nous avons aussi essayé de mettre en rapport l'histoire de la procédure et l'histoire de la démonologie, alors que l'approche juridique et l'approche théologique sont souvent séparées par la force des choses, relevant de deux spécialités très différentes. Il n'y a pas d'interprétation univoque du diable, mais une diversité d'enjeux lisibles à travers cette figure. C'est aussi pour cette raison que nous avons donné une place à l'enquête historiographique. Comme Carmen Rob-Santer le montre dans son article, l'historiographie allemande a davantage utilisé le Marteau des sorcières dans un sens idéologique qu'elle ne l'a examiné dans le détail comme un objet historique, et les connaissances à propos de ce texte si célèbre sont encore lacunaires et contradictoires. De même, Georg Modestin montre dans les pages qu'il consacre à Reginald Scot que dans certains cas, les concepts élaborés par les historiens pour comprendre le mécanisme des chasses aux sorcières reprennent des schémas narratifs déjà contenus dans les traités de démonologie : le motif de la charité refusée est ainsi davantage un topos littéraire que l'analyse d'une quelconque réalité. Ces deux articles rappellent que l'histoire de la sorcellerie est aussi traversée par les grands courants de l'historiographie, au fil des modes et des tendances. Elle l'est d'ailleurs peut-être plus que d'autres, car la sorcellerie est avant tout un objet de discours: qu'est-ce, " réellement ", qu'une sorcière?

Démonologie et répression de la sorcellerie : les rythmes d'une histoire pluriséculaire

Les textes réunis ici tentent de répondre à certaines de ces interrogations, tout en proposant de nouvelles pistes de réflexion. On peut les regrouper autour de trois moments. Le premier, celui de la genèse durant les $\mathrm{XIII}^{\mathrm{e}}$ et $\mathrm{xIV}^{\mathrm{e}}$ siècles des outils intellectuels utilisés plus tard pour la chasse aux sorcières, est abordé par l'article d'Alain Boureau sur Jean XXII (1316-1334). C'est en effet ce pape qui, un siècle avant le déclenchement de la chasse aux sorcières, a posé les bases d'une nouvelle perception de la magie et des savoirs qui ont pu lui être associés, comme l'astrologie ou l'alchimie. La bulle Super illius specula (1326 ou 1327) de Jean XXII énonce pour la première fois que les pratiques magiques (fabrication d'images, d'anneaux ou de miroirs) dérivent directement de l'invocation des démons et que, de fait, les personnes qui se livrent à de tels actes encourent les peines réservées aux hérétiques. La magie, mais aussi indirectement les sortilèges, sont dès lors pensés comme des hérésies, et ceux qui sont soupçonnés de s'y livrer peuvent tomber dans le ressort de l'inquisition. Les rapports avec les démons entrent dans le champ des faits réels et ne sont plus des illusions diaboliques, tels les rêves de vol nocturne du vieux canon Episcopi ${ }^{2}$. La définition de Jean XXII du fait hérétique (factum hereticale) a des conséquences judiciaires, puisqu'elle met en avant la nécessité d'une enquête (inquisitio) rapide et efficace, au détriment de la procédure accusatoire. Dans cette nouvelle définition, l'hérésie n'est plus seulement une affaire d'opinion ou de croyance erronée : elle se manifeste par des actions - elle fait quelque chose. C'est bien sur ce terreau que se développe le sabbat des sorcières dans les années 1430: pensons aux efforts continus des inquisiteurs et des démonologues pour chercher à tout prix - et en vain - des preuves matérielles de la réalité des rencontres sabbatiques. Comme le démontre ici Alain Boureau, la bulle de Jean XXII n'est qu'un élément parmi un large ensemble de documents qui atteste les préoccupations du pape pour la magie et les invocations de démons, qu'il s'agisse de consultations, d'expertises, de demandes d'action judiciaire ou de mises sur pied de 
commissions d'enquête spéciales. Toutes ces actions ont pour objectif de dissiper l'ambiguïté des attitudes relatives à la magie, mais aussi à l'astrologie et à l'alchimie, ce qui n'est pas sans poser problème car alchimistes et astrologues ont noué des liens étroits avec les cours pontificales et princières.

11 Si la doctrine contre les magiciens et les sorciers semble être prête vers 1330, pourquoi n'est-ce qu'un siècle plus tard que l'on rencontre dans les textes les premières sectes de sorciers adorateurs du diable, infanticides et cannibales? La question a souvent été posée ; les contributions rassemblées ici proposent de nouvelles réponses. Pour Alain Boureau, ce « retard à l'allumage » serait dû à la réticence de la papauté à déléguer son pouvoir d'enquête à l'inquisition. C'est « l'abandon forcé de l'absolutisme pontifical, après les conciles de Constance et de Bâle, qui ouvrit les premières campagnes judiciaires contre les sorciers et les adorateurs de démons ». On sait maintenant à quel point les conciles de Constance et de Bâle ont été des lieux d'échanges féconds au sujet des hérétiques au sens large, qu'il s'agisse des Hussites, des hérésies du Libre Esprit et des sectes de sorciers démoniaques, mais aussi de l'ensemble des phénomènes surnaturels, dont on questionne la nature divine ou démoniaque, comme les visions, les miracles, les extases, les possessions, etc.; la question des rituels et des sacrements participe aussi de cette redéfinition des rapports avec le divin.

On en arrive au second moment de notre chronologie, celui de la cristallisation de la sorcellerie et de la démonologie, au cours $\mathrm{du} \mathrm{xv}^{\mathrm{e}}$ siècle. Il semble donc qu'une fois réglé le problème de l'ambiguïté de la magie, celle-ci entrant dans la catégorie de l'hérésie, l'ambivalence s'attache à d'autres phénomènes impliquant le surnaturel, pouvant même conduire à une mise en doute des miracles ou des visions - pensons là à Jeanne d'Arc. À ce titre, la figure du réformateur Jean Nider, que l'on retrouve à la fois aux conciles de Constance et de Bâle, est exemplaire: Gábor Klaniczay met en valeur l'originalité du schéma conceptuel du dominicain, qui juxtapose dans son Formicarius des œuvres d'origine céleste ou diabolique, en tentant d'en déterminer à chaque fois la nature. Nider fait l'éloge des femmes mystiques ou des "saintes vivantes", telle Catherine de Sienne, mais dénonce les simulatrices ou fausses prophétesses. C'est dans ce cadre élargi qu'il faut saisir les premiers récits de sectes cannibales et démoniaques rapportés - élaborés ? - par Nider relatifs à la Suisse, ainsi que les épisodes se rapportant au sorcier Scaedeli, adepte de magie noire. Gábor Klaniczay tente de montrer la pertinence de la notion anthropologique de "chamanisme " pour réussir à prendre en compte tous les types de communications avec le surnaturel, qu'ils soient divins ou démoniaques, et pour penser dans un même cadre conceptuel le saint et le sorcier, avec comme point commun l'idée de «transe » et la présence d'un médiateur, un "chamane", vivant au sein de la communauté afin de répondre à ses préoccupations quotidiennes.

13 L'évolution simultanée de la sainteté et de la sorcellerie au cours des $\mathrm{XIV}^{\mathrm{e}}$ et $\mathrm{XV}^{\mathrm{e}}$ siècles pourrait avoir produit ce qu'André Vauchez ${ }^{3}$ désignait comme la naissance du soupçon à l'égard des phénomènes surnaturels, avec pour conséquence une exigence, de la part tant de la population que des autorités, de preuves manifestes et tangibles de la réalité des manifestations divines ou démoniaques. Stigmates (contestés) de sainte Catherine ou cicatrices de brûlures endurées en enfer par une femme de Bourg-en-Bresse (Gábor Klaniczay), mais aussi marques diaboliques et maladies des femmes après un coït satanique dévoilées en cours de procédure inquisitoire : le besoin de faits éprouvé par Jean XXII vers 1330 semble un siècle plus tard partagé par tous et dans des domaines 
aux frontières de plus en plus incertaines. Rappelons aussi que ce sont souvent les mêmes personnages qui traitent du discernement des esprits - soit de la manière de différencier les bons et les mauvais esprits, les anges et les démons - et qui ont par ailleurs affaire aux visions des saintes et aux opérations démoniaques : pour exemple, mentionnons Henri de Langenstein, Henri de Freimar, Jean Gerson, Jean Nider, ou encore Nicolas Jacquier. Les démonologues de la fin du Moyen Âge sont en premier lieu des théologiens.

Ce jeu de miroir entre les deux pôles du panthéon surnaturel ne se confine pas aux élites religieuses. Des artistes mettent en scène et jouent sur cette ambivalence, comme le montre ici Franck Mercier. Les manuscrits du Traité du crisme de Vauderie de Jean Taincture (Tinctoris) offrent vers 1460-1470 l'une des premières représentations du sabbat des sorcières, à travers trois miniatures qui déclinent le même thème. Un bouc trône au milieu de l'image, entouré de fidèles agenouillés à ses pieds, les mains jointes ou tenant des cierges allumés; l'un des adeptes du culte démoniaque embrasse l'animal sur le postérieur, alors que le ciel est rempli de démons ailés amenant les sorciers au sabbat. Cette cérémonie, si elle dit le sabbat, reflète aussi une étrange solennité liturgique : le sabbat apparaît au spectateur comme une messe détournée - et on peut rappeler ici que la première utilisation du mot «liturgie » en français est due à Jean Bodin, démonologue et politologue de la seconde moitié du xvI siècle, précisément au sujet du sabbat. Mais, comme le suggère Franck Mercier, les trois miniatures des manuscrits de Taincture semblent subvertir un autre schéma iconographique majeur : celui de l'adoration de l'Agneau christique, dont Jan et Hubert Van Eyck ont donné en 1432 une interprétation magistrale dans le retable de Gand. Bouc ou agneau, démon ou Christ, les images agissent en trompe-l'œil en détournant des symboles christiques. $\mathrm{Si}$, dans cette perversion d'image, la position centrale du bouc souligne la Majesté du démon - il est le "prince des ténèbres "-, celui-ci est montré aussi comme un usurpateur de la majesté divine qu'il copie maladroitement. Le traité du théologien de Cologne Jean Taincture ne dit pas autre chose : il ne cesse de mettre en garde contre les illusions du diable, qui ne sont que des simulacres imparfaits de la Création divine.

C'est bien l'ampleur de ces illusions diaboliques qui pose problème aux démonologues. Certes, le diable est le grand illusionniste, mais qu'en est-il des «synagogues » de sorciers? Sont-elles réelles ou s'agit-il de rêves instillés par les démons dans les esprits des hommes? Et comment peut-on mener une action judiciaire, torturer et condamner... pour un rêve? C'est dans cette ambivalence qu'il faut lire l'«affaire Guillaume Adeline » (Martine Ostorero), du nom d'un prédicateur bénédictin qui, sous contrainte du diable, aurait affirmé publiquement que le sabbat n'était qu'un songe. Comme le personnage est lui-même soupçonné d'appartenir à une secte de sorciers, on comprend les enjeux de l'affaire, qui tombe à point nommé pour servir les partisans de la thèse d'un sabbat réel. Les démonologues français du $\mathrm{Xv}^{e}$ siècle se servent largement de ce cas pour étayer leur argumentation, alors que la question ne semble pas se poser avec la même acuité en Italie ou dans l'Empire. Une différence culturelle qui mérite d'être relevée, même si elle ne trouve pas d'explication claire pour l'heure.

On arrive enfin au troisième moment dans cette histoire de la sorcellerie et de la démonologie, celui de la propagation et de l'acculturation du thème lors des $\mathrm{XVI}^{\mathrm{e}}$ et $\mathrm{XVII}^{\mathrm{e}}$ siècle, jusqu'à son extinction. Le savoir moderne s'appuie alors sur les constructions médiévales en les réinterprétant par le biais de transmissions culturelles complexes, croisant magie et sorcellerie. Ainsi, les contributions consacrées au Moyen Âge 
présentent des démons aux multiples visages : ceux rencontrés aux sabbats nocturnes, sous l'apparence d'homme noir, de bouc, de chat ou d'autre animal noir, sont multiformes (Franck Mercier, Martine Ostorero). Mais en quoi ressemblent-ils aux esprits maléfiques du début $d u x I^{e}$ siècle, que l'apprenti magicien peut invoquer, choisissant dans un "bottin mondain démoniaque » (Jean-Patrice Boudet) le démon de son choix suivant sa puissance, sa fonction, le volume de ses troupes, comptabilisés en légions? Les uns entraînent les hommes à commettre le plus de mal possible sur la Terre, à s'en prendre aux hommes, aux femmes, aux enfants, à bafouer l'Église, voire le pouvoir politique, et les persuadent de commettre des actes sexuels avec lui ; les autres, à l'apparence de monstres, hybrides d'homme et d'animaux, s'ils sont conjurés convenablement, offrent au magicien des capacités intellectuelles hors du commun et leur permettent d'obtenir des richesses, de rencontrer l'amour, la grâce et la guérison. Et pourtant, ces démons portent les mêmes noms : Satan, Lucifer, Bélial, Belzébuth se retrouvent autant au sabbat que dans les livres de magie, mais avec un statut différent. En outre, les démons qui hantent Jean XXII se dissimulent dans des images de cire ou de plomb, à caractère magique, qui paraissent se multiplier autour du souverain pontife, le poussant à redéfinir les rapports entre la magie et l'hérésie (Alain Boureau). L'omniprésence et l'extrême visibilité des démons, qui envahissent presque tout l'espace du monde chrétien, s'estompe par la suite. À la fin du xvi siècle, chez l'anglais Reginald Scot, le diable est invisible et purement spirituel, ne conservant qu'une dimension morale et métaphorique, permettant par là même à Scot de s'en moquer et de tourner en ridicule le sabbat, qui ne fera qu'une avancée timide dans les terres anglaises au XVII ${ }^{e}$ siècle (Georg Modestin). Avec Johannes Althusius, le diable devient la métaphore, simple mais efficace, de tous les crimes commis contre la société civile. Stuart Clark a montré lors du débat de décembre 2000 les rapports entre la théorie politique et la démonologie, à partir de Johannes Althusius et de Johann Georg Godelmann. En 1591, ce dernier, alors qu'il publie ses conférences sur la Caroline dans son Tractatus de magis, veneficis et lamiis deque his recte cognoscendis et puniendis, se plaint d'abus légaux et d'erreurs judiciaires commis en Allemagne en matière de sorcellerie. Or, Johannes Althusius, auteur de la Politica methodice digesta, l'un des ouvrages les plus lus sur la théorie politique au début du XVII e siècle, reprend les positions de Godelmann dans une allocution intitulée Ad judicem admonitio, publiée à la fin de la première partie du Tractatus de Godelmann. La juxtaposition de points de vue identiques exprimés par deux intellectuels, dont l'un est considéré habituellement comme un démonologue et l'autre comme un théoricien du politique, souligne la grande affinité existant entre la démonologie modérée exprimée par ces deux hommes et la philosophie politique constitutionnelle rendue fameuse par Althusius.

Les différences repérables, du Moyen Âge à la première modernité, entre le traitement de la magie et celui de la sorcellerie, incitent finalement à faire une histoire politique du diable : Jean XXII s'intéresse à la magie parce qu'il se sent l'objet de rites occultes orientés contre lui, dans un contexte où les clans (Spirituels, Orsini, Colonna, Visconti) s'affrontent. Les grandes affaires politico-religieuses du xIV siècle (procès des Templiers, de Boniface VIII, de Guichard de Troyes, de Bernard Délicieux, d'Enguerrand de Marigny, etc.) ont toutes un lien avec le diable, l'envoûtement, les maléfices ou encore la magie rituelle. Comme le souligne Jean-Patrice Boudet, les textes de magie sont de véritables défis à l'égard des autorités de l'Église et de l'État, car ils offrent des potentialités énormes à ceux qui invoquent les démons : pouvoir, richesse et savoir. Au moment des conciles de Constance et de Bâle, c'est peut-être entre conciliaristes et 
partisans de la monarchie pontificale que la vision du diable et la position par rapport au sabbat est la plus différente; Guillaume Adeline ne fait-il pas les frais, sur sa propre personne, de deux conceptions qui s'opposent et dont les enjeux semblent aussi politiques? Aux dires du chroniqueur lucernois Hans Fründ qui décrit les premières chasses aux sorcières en Valais vers 1428 , les sectes de sorciers sont des formes de sociétés secrètes suspectées de fomenter des complots dans la clandestinité pour renverser le pouvoir établi. Enfin, Jean Taincture, en dénonçant la puissance usurpatrice du Diable et de tous ceux qui se risquent à le suivre, entend rétablir l'obéissance et le respect de l'ordre divin, au moment même où les troubles liés à la Vauderie d'Arras font chanceler les pouvoirs en place. De l'anthropologie chrétienne, le diable nous ramène donc, par le biais des constructions étatiques, à notre point de départ, les transformations du lien entre savoirs et pouvoirs.

Reste à ne pas oublier que derrière les discours érudits, la sorcellerie est avant tout "une histoire qui tue», selon la formule de Georg Modestin. Notre activité d'entomologistes du devenir occidental et notre focalisation sur les sources savantes ne doit pas effacer la réalité sociale de la répression judiciaire, connue par les très nombreuses sources de la pratique concernant la mise en accusation et l'exécution des victimes. Sabbat, sorcellerie, diable et démons sont le fruit de discours savants dont la répétition par les juges et les prédicateurs, à la manière des prophéties autoréalisatrices, a fini par créer du réel, provoquant la mort d'hommes et de femmes sur les bûchers de l'Occident, et suscitant peut-être d'étranges pratiques dans les campagnes du $\mathrm{Xv}^{\mathrm{e}}$ et $\mathrm{XVI}^{\mathrm{e}}$ siècles :

une bonne partie des victimes du Marteau des sorciers [sic] et autres traités rédigés par des démonologues surexcités et lus assidûment par les juges étaient à coup sûr de pauvres hères inoffensifs qui s'étaient attiré l'antipathie des voisins par un air ou des façons bizarres, des quintes d'humeur, le goût de la solitude ou quelque autre caractéristique peu goûtée des gens (...). Mais il faut aussi compter avec ceux qu'une malignité véritable, une vague rancune contre les misères et les brimades subies, un goût décrié ou un besoin inassouvi menaient au sabbat en fait ou en songe. Après les journées passées à biner les champs de navets ou à piocher dans des tourbières, des gueux trouvaient dans le petit groupe dépenaillé, accroupi dans un hallier autour d'un tas de braises, l'équivalent de nos danses redevenues primitives, de nos musiques de grincements et de cris, peut-être de nos fumées et de nos potions hallucinatoires. Ils y satisfont l'instinct de s'agglomérer comme des larves; ils goûtent la chaleur et la promiscuité des corps, la nudité, interdite ailleurs, le petit frisson ou le petit ricanement de l'ignoble ou de l'illicite. Le reflet des flammes qui joue sur ces misérables ne présage pas seulement la mort patibulaire, toujours préparée pour eux ; ces lueurs viennent du fond d'eux-mêmes, sinon d'un autre monde 4

BIBLIOGRAPHIE

J. HANSEN, Quellen und Untersuchungen zur Geschichte des Hexenwahns und der Hexenverfolgung im Mittelalter, Bonn, 1901 (reprint Hildesheim, 1963). 
L'Imaginaire du sabbat. Édition critique des textes les plus anciens (1430 ca-1440 ca), réunis par M. Ostorero, A. PARAVicini BAgliani et K. UtZ TRemP, avec la collaboration de C. ChÈne, Lausanne, 1999 (CLHM, 26).

W. BEHRINGER, G. JEROUSCHEK, W. TSCHACHER (éd.), Heinrich Kramer (Institoris). Der Hexenhammer. Malleus Maleficarum, Munich, 2000.

A. DANET, Henry Institoris (Kraemer). Jacques Sprenger. Le Marteau des Sorcières. Malleus Maleficarum. Traduit du latin et précédé de « L'Inquisiteur et ses sorcières », Grenoble, 1990.

Sur la sorcellerie

R. BRIGGS, Witches \& Neighbours. The Social and Cultural Context of European Witchcraft, Londres, 1997.

L. CARO BAROJA, Les Sorcières et leur monde, Paris, 1978.

J. ChIFfoleau, « Dire l'indicible : remarques sur la catégorie du nefandum du XII ${ }^{\mathrm{e}}$ au XV siècle », Annales ESC, 45, mars-avril 1990, p. 289-324.

S. CLARK, Thinking with Demons. The Idea of Witchcraft in Early Modern Europe, New York, 1997.

N. CoHN, Démonolâtrie et sorcellerie au Moyen Âge. Fantasmes et réalités, Paris, 1982.

C. GINZBURG, Le Sabbat des sorcières, Paris, 1992.

S. HOUDARD, Les Sciences du Diable. Quatre discours sur la sorcellerie (XVe-XVII siècle), Paris, 1992.

R. KIECKHEFER, European Witch Trials : their Foundations in Popular and Learned Culture, 1300-1500, Londres, 1976.

Languages of Witchcraft. Narrative, Ideology and Meaning in Early Modern Culture, éd. par S. CLARK, Londres, 2001.

Le Sabbat des sorciers en Europe (XVe-XVIII siècle), éd. par N. JACQUES-CHAQUIN et M. PRÉAUD, Grenoble, 1993.

«Les sorcières, les seigneurs et les juges. La persécution des sorciers et sorcières dans le territoire de la Suisse actuelle à la fin du Moyen Âge ", dossier édité par G. MODESTIN et K. UTZ TREMP, Revue Suisse d'Histoire, 52, 2002, p. 103-162. [Bilan des travaux sur la sorcellerie dressé par les historiens suisses, assorti d'une bibliographie complète.]

B. P. LEVACK, La Grande Chasse aux sorcières en Europe aux débuts des Temps Modernes, Seyssel, 1991.

R. MUCHEMBLED, La Sorcière au village, Paris, 1979.

R. MuchEMBLED, Le Roi et la sorcière. L'Europe des bûchers, XV'-XVII siècles, Paris, 1993.

J.-C. SchmitT, « Les superstitions », dans Histoire de la France religieuse, dir. par J. LE GOFF, R. RÉMOND, t. 1, Paris, 1988, p. 417-551.

K. THомAs, Religion and the Decline of Magic. Studies in Popular Beliefs in Sixteenth-and SeventeenthCentury England, Londres, 1971.

H. R. TREVOR-ROPER, « L'épidémie de sorcellerie en Europe », dans ID., De la Réforme aux Lumières, Paris, 1972

Sur la magie

J.-P. BOUDET, « Les condamnations de la magie à Paris en 1398 », Revue Mabillon, nouvelle série, 12 (t. 73), 2001, p. 121-157.

R. KIECKHEFER, Magic in the Middle Ages, Cambridge, 1989. 
R. KIECKHEFER, Forbidden Rites. A Necromancer's Manual of the Fifteenth Century, Stroud, 1997.

Les Anges et la Magie au Moyen Âge (8-9 décembre 2000), Rome, 2002 (MEFRM, à paraître).

N. WeILL-PAROT, Les «Images astrologiques » au Moyen Âge et à la Renaissance. Spéculations intellectuelles et pratiques magiques ( $\mathrm{XII}^{e}-\mathrm{XV}^{e}$ siècle), Paris, 2002.

Sur le diable et les démons

J. BASCHET, Les Justices de l'au-delà. Les représentations de l'enfer en France et en Italie (XII ${ }^{e}-\mathrm{XV}^{e}$ siècle), Rome, 1993.

R. MuCHEMBLED, Une histoire du Diable (XII ${ }^{e}-X^{e}$ siècle), Paris, 2000.

J. B. RusSEL, Lucifer. The Devil in the Middle Ages, Ithaca-Londres, 1984.

\section{NOTES}

1. Ce numéro est issu d'une rencontre tenue le 15 décembre 2000 à l'Institut Historique Allemand de Paris. Nous remercions son directeur, M. Werner Paravicini, qui a eu l'amabilité et la générosité d'accueillir cet atelier international. À cette occasion, Jacques Chiffoleau a eu la gentillesse de dire quelques mots de conclusion et nous l'en remercions également. La contribution que présenta alors Stuart Clark, auteur de Thinking with demons (New York 1997), vient de paraître en anglais sous le titre : "Johannes Althusius and the Politics of Witchcraft ", dans L. M. Anderson et al. (eds), Rätten : En Festskrift till Bengt Ankarloo, Lund, 2000, p. 272-290. Nous remercions enfin Dominique Iogna-Prat, Bruno Laurioux et Valérie Theis pour leur relecture amicale de ces pages.

2. Ce texte de loi rédigé vers 900 et inséré au XII ${ }^{\mathrm{e}}$ s. dans le Décret de Gratien rejette du coté de l'illusion et de la superstition la croyance selon laquelle des femmes scélérates, sous l'emprise des démons, se déplaceraient réellement dans les airs à la suite de Diane, la déesse païenne.

3. A. Vauchez, «La naissance du soupçon : vraie et fausse sainteté aux derniers siècles du Moyen Âge ", dans Id., Saints, prophètes et visionnaires. Le pouvoir surnaturel au Moyen Âge, Paris, 1999, p. 208-219.

4. M. Yourcenar, Archives du Nord, Paris, 1991, p. 990.

\section{AUTEURS}

\section{MARTINE OSTORERO}

Université de Lausanne, Section d'Histoire médiévale, BFSH 2, CH-1015 Lausanne

\section{ÉTIENNE ANHEIM}

École française de Rome, 67 Piazza Farnese, I-00186 Rome 